\title{
artigo
}

\section{Repercussões psicopatológicas em enfermagem decorrentes da pandemia do COVID-19: uma revisão integrativa}

\author{
Psychopathological repercussions in nursing arising out of the COVID-19 pandemic: an integrative review \\ Repercusiones psicopatológicas en enfermería derivadas de la pandemia COVID-19: una revisión integrativa
}

\begin{abstract}
RESUMO
Objetivo: identificar as repercussões psicopatológicas em profissionais da enfermagem decorrentes da pandemia do covid-19. Método: Trata-se de uma revisão integrativa da literatura realizada entre o período de outubro de 2020 a janeiro de 2021, onde foram analisados artigos publicados nas plataformas de bases de dados Google Acadêmico e Biblioteca virtual em Saúde (BVS). Foram adotados como critérios de inclusão: publicações em português, artigos originais e de livre acesso, indexados entre dezembro de 2019 e janeiro de 2021. Resultados: a amostra final desta revisão foi constituída por 06 artigos, selecionados pelos critérios de inclusão previamente estabelecidos. Conclusão: diante ao exposto, percebe-se que os profissionais de enfermagem na linha de frente que estão diretamente envolvidos no diagnóstico, tratamento e cuidado de pacientes com COVID-19 correm o risco de desenvolver sofrimento psicopatológico comprometendo assim sua saúde mental e por consequência sua vida e a assistência que presta aos pacientes.
\end{abstract}

DESCRITORES: COVID-19; Saúde mental; Enfermagem; Psicopatologia.

\section{ABSTRACT}

Objective: to identify the psychopathological repercussions in nursing professionals resulting from the covid-19 pandemic. Method: This is an integrative review of the literature carried out between October 2020 and January 2021, where articles published on the Google Scholar and Virtual Health Library (VHL) database platforms were analyzed. Inclusion criteria were: publications in Portuguese, original and open access articles, indexed between December 2019 and January 2021. Results: the final sample of this review consisted of 06 articles, selected by the inclusion criteria previously established. Conclusion: in view of the above, it is clear that nursing professionals in the front line who are directly involved in the diagnosis, treatment and care of patients with COVID-19 are at risk of developing psychopathological suffering, thus compromising their mental health and consequently their life and assistance to patients.

DESCRIPTORS: COVID-19; Mental health; Nursing; Psychopathology.

\section{RESUMEN}

Objetivo: Identificar las repercusiones psicopatológicas en los profesionales de enfermería derivadas de la pandemia del covid-19. Método: Se trata de una revisión integradora de la literatura realizada entre octubre de 2020 y enero de 2021, donde se analizaron los artículos publicados en las plataformas de base de datos Google Scholar y Virtual Health Library (BVS). Los criterios de inclusión fueron: publicaciones en portugués, artículos originales y de acceso abierto, indexados entre diciembre de 2019 y enero de 2021. Resultados: la muestra final de esta revisión estuvo conformada por 06 artículos, seleccionados por los criterios de inclusión previamente establecidos. Conclusión: en vista de lo anterior, es claro que los profesionales de enfermería de primera línea que están directamente involucrados en el diagnóstico, tratamiento y cuidado de los pacientes con COVID-19 están en riesgo de desarrollar sufrimiento psicopatológico, comprometiendo así su salud mental y consecuentemente. su vida y asistencia a los pacientes.

DESCRIPTORES: COVID-19; Salud mental; Enfermería; Psicopatología.

RECEBIDO EM: 30/01/2021 APROVADO EM: 15/02/2021

\section{Lina Eduarda Silva Costa}

Graduanda em enfermagem pela Faculdade Carajás. PA-Brasil.

ORCID: 0000-0002-9992-4881 


\section{Fernanda Vale de Oliveira}

Graduanda em enfermagem pela Faculdade Carajás. PA-Brasil.

ORCID: 0000-0002-1677-1876

\section{Anne Gabrielle Moreira Carneiro}

Graduanda em enfermagem pela Faculdade Carajás. PA-Brasil.

ORCID: 0000-0003-0953-5493

\section{Samara de Araújo Alves Ribeiro}

Graduanda em enfermagem pela Faculdade Carajás. PA-Brasil.

ORCID: 0000-0002-8439-1904

\section{Amanda Cristina de Souza Barros}

Graduanda em enfermagem pela Faculdade Carajás. PA-Brasil.

ORCID: 0000-0003-3647-5748

\section{Paula Paulina Costa Tavares}

Enfermeira. Mestrado Profissional em Promoção da saúde pelo Centro universitário Adventista de São Paulo. Docente do curso de Enfermagem da Faculdade Adventista da Bahia. BA-Brasil.

ORCID: 0000-0002-9275-8884

\section{INTRODUÇÃO}

$\mathbf{N}$ o dia 30 de janeiro de 2020 a Organização Mundial da Saúde (OMS) declarou que o Corona Virus Disease 2019 (COVID-19) se tornara uma Emergência de Saúde Pública de Importância Internacional (ESPII) - caracterizado como o mais alto nível de alerta da Organização, pouco depois em 11 de março de 2020 a OMS declarou que o COVID-19 tornou-se uma pandemia ${ }^{(1)}$.

No Brasil, ainda em março de 2020, foram confirmados casos e óbitos na região sudeste decorrentes do coronavírus, e desde então, os profissionais de enfermagem estão na linha de frente dos cuidados com os indivíduos acometidos pela doença. No Ano Internacional da Enfermagem e Obstetrícia, como reconhecimento ao papel imprescindível na prestação de serviços de saúde, ficou ainda mais evidente face aos desafios enfrentados por causa da pandemia, que esses profissionais precisam demonstrar competências como conhecimento técnico-científico, habilidade e controle emocional para lidar com a prática assistencial, mesmo com todo o desgaste decorrente da pandemia e da responsabilidade com a vida dos pacientes ${ }^{(2,3)}$.

Incontestavelmente, o trabalho em períodos de surtos, pode acarretar problemas que afetam a saúde mental dos profissio- nais, sendo que tais problemas são mais perceptíveis em profissionais atuantes em unidades de alto risco. ${ }^{(3)} \mathrm{A}$ enfermagem tem tido grande destaque nesse período de enfrentamento a pandemia, contudo para além do destaque a profissão encontra-se em alto grau de exposição e a riscos de contaminação, violências e impactos na saúde mental ${ }^{(4)}$. Com base em Huang, et al $(2020)^{(5)}$ e observando este cenário, é possível constatar que a pandemia do COVID-19 tem sido uma fonte de grande estresse para os indivíduos e grupos sociais, visto que, os seres humanos experimentam distintos níveis de crises emocionais. Entretanto, é especialmente mais difícil para aqueles que estão atuando no centro da crise.

Diante disto, este estudo tem como questão norteadora: Quais as repercussões psicológicas e emocionais decorrentes da pandemia do covid-19 para os profissionais da enfermagem? O objetivo deste estudo foi identificar as repercussões psicopatológicas em profissionais da enfermagem decorrentes da pandemia do covid-19.

\section{MÉTODO}

Trata-se de uma revisão integrativa da literatura realizada entre outubro de 2020 e janeiro de 2021, onde foram analisados artigos publicados nas plataformas de bases de dados Google Acadêmico e Biblioteca virtual em Saúde (BVS). Foram adotados como critérios de inclusão: publicações em português ou inglês, artigos originais e de livre acesso, indexados entre dezembro de 2019 e janeiro de 2021 (considerando o período de surgimento da patologia). Foram utilizados os seguintes Descritores de Ciências da Saúde (DeCS): COVID-19; Enfermagem; Saúde mental; Psicopatologia e seus correspondentes em inglês: COVID-19; Nursing; Mental health; Psychopathology. Para tanto utilizou-se dos operadores booleanos and, or. Os critérios de exclusão foram: artigos duplicados, de acesso restrito, teses, monografias e resumos de anais.

Esta revisão seguiu as seguintes etapas: 1) Elaboração do problema da pesquisa; 2) Busca nas bases de dados com aplicação dos critérios de inclusão e exclusão; 3) Leitura e análise crítica dos artigos; 4) Categorização e organização dos achados; 5) Interpretação e discussão dos resultados; 6) concluído mediante apresentação dos resultados e análise crítica dos dados obtidos pertinentes à pesquisa de forma descritiva.

\section{RESULTADOS}

A partir dos achados nas plataformas de bases de dados mencionadas, proce- 


\section{artigo}

Costa, L.E.S.; Oliveira, F.V.; Carneiro, A.G.M.; Ribeiro, S.A.A.; Barros, A.C.S.; Tavares, P.P.C.

Repercussões psicopatológicas em enfermagem decorrentes da pandemia do COVID-19: uma revisão integrativa

Figura 1. Fluxograma das informações da pesquisa nas bases de dados mapeando o número de registros identificados, incluídos e excluídos, e os motivos para exclusões. Marabá, PA, Brasil, 2021.

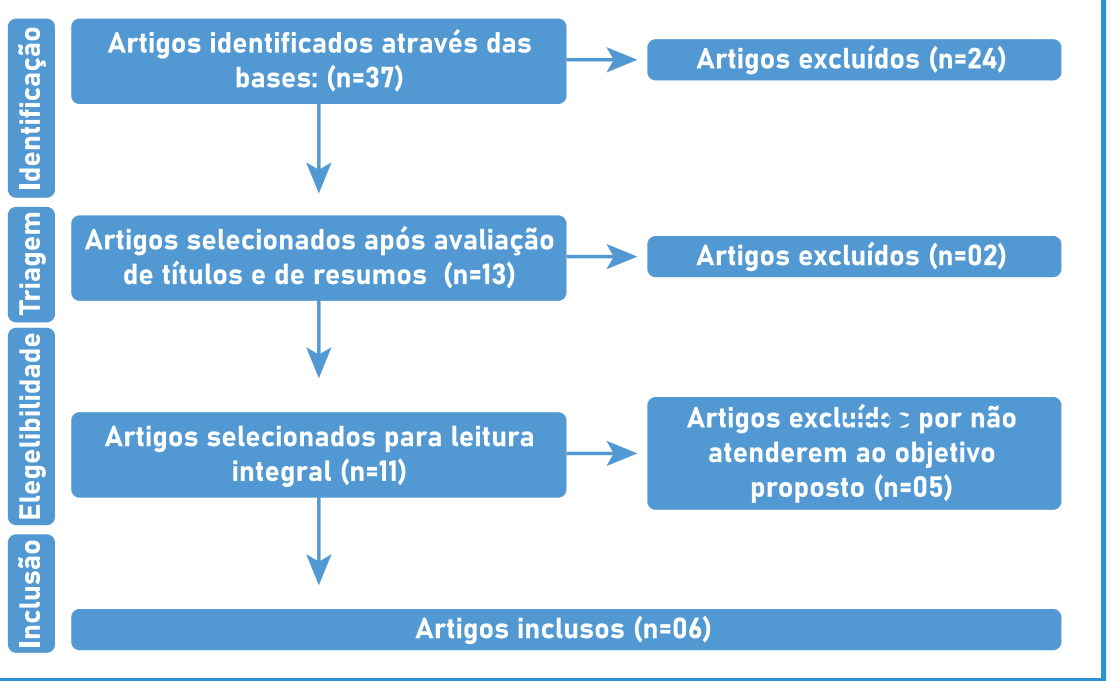

deu-se a leitura dos títulos de 37 artigos, foram selecionados aqueles que melhor atendiam a temática proposta, considerando os descritores. Em seguida foi realizada a leitura dos resumos, resultando em 13 estudos que atenderam aos critérios de inclusão. Após leitura completa e minuciosa, 06 artigos compuseram o corpus dessa revisão. Os dados obtidos foram organizados e analisados de forma crítica comparando-os com a literatura científica.

A amostra final desta revisão foi constituída por 06 artigos, selecionados pelos critérios de inclusão previamente estabelecidos. O Quadro 1 apresenta as especificações de cada artigo, sendo os respectivos: autores, título, principais achados, revista e ano.

\section{Quadro 1. Detalhamento dos estudos selecionados. Marabá, PA, Brasil, 2021.}

\begin{tabular}{|c|c|c|c|c|}
\hline $\mathbf{N}^{\circ}$ & AUTORES & TÍTULO & PRINCIPAIS ACHADOS & REVISTA/ANO \\
\hline 01 & $\begin{array}{l}\text { Ramos-Toescher } \\
\text { AM, Tomas- } \\
\text { chewisk-Barlem } \\
\text { JG, Barlem ELD, } \\
\text { Castanheira JS, } \\
\text { Toescher RL. }{ }^{(6)}\end{array}$ & $\begin{array}{l}\text { Saúde mental de profis- } \\
\text { sionais da enfermagem } \\
\text { durante a pandemia da } \\
\text { cOVID-19: recursos de } \\
\text { apoio. }\end{array}$ & $\begin{array}{l}\text { Em decorrência da pandemia do novo coronavírus, interven- } \\
\text { ções psicológicas voltadas para os profissionais de enfer- } \\
\text { magem vêm desempenhando um papel extremamente } \\
\text { importante para configuração do atual cenário. }\end{array}$ & $\begin{array}{c}\text { Esc. Ana Nery. } \\
2020\end{array}$ \\
\hline 02 & $\begin{array}{l}\text { Pereira MD, Torres } \\
\text { EC, Pereira MD, } \\
\text { Antunes PFS, Costa } \\
\text { CFT. }{ }^{(7)}\end{array}$ & $\begin{array}{l}\text { Sofrimento emocional } \\
\text { dos enfermeiros no } \\
\text { contexto hospitalar } \\
\text { frente à pandemia de } \\
\text { COVID-19 }\end{array}$ & $\begin{array}{l}\text { O estado de calamidade global no âmbito da saúde } \\
\text { tem causado agravos na saúde mental principalmente } \\
\text { dos enfermeiros, visto que, são expostos a risco de } \\
\text { contaminação, falta de materiais, altas demandas, } \\
\text { experiências intensas como dores, sofrimentos e } \\
\text { morte árdua que gera sentimentos de estresse, tensão, } \\
\text { nervosismo, fadiga, agressividade, episódios de pânico e } \\
\text { até depressão. }\end{array}$ & $\begin{array}{c}\text { Research, } \\
\text { Society and } \\
\text { Development. } \\
2020\end{array}$ \\
\hline 03 & $\begin{array}{c}\text { Souza NVDO, } \\
\text { Carvalho EC, Soares } \\
\text { SSS, Varella TCMM, } \\
\text { Pereira SEM, An- } \\
\text { drade KBS. }{ }^{(8)}\end{array}$ & $\begin{array}{l}\text { Trabalho de enfermagem } \\
\text { na pandemia da Covid-19 } \\
\text { e repercussões para a } \\
\text { saúde mental dos traba- } \\
\text { Ihadores }\end{array}$ & $\begin{array}{l}\text { A escassez de equipamento de proteção individual, fragilida- } \\
\text { de na descrição dos protocolos e dos fluxos para o controle } \\
\text { efetivo de infecções, as prolongadas horas de trabalho, a } \\
\text { formação profissional inadequada para o cenário de crise e } \\
\text { as incertezas em relações as medidas terapêuticas. Marcan- } \\
\text { do assim um cenário que eleva o potencial de impacto nega- } \\
\text { tivo na saúde mental dos trabalhadores de enfermagem. }\end{array}$ & $\begin{array}{l}\text { Rev Gaúcha } \\
\text { Enferm. } 2021\end{array}$ \\
\hline 04 & $\begin{array}{c}\text { Portugal JKA, Reis } \\
\text { MHS, Barão EJS, } \\
\text { Souza TTG, Guima- } \\
\text { rães RS, Almeida } \\
\text { LS, et al. }{ }^{(9)}\end{array}$ & $\begin{array}{l}\text { Percepção do impacto } \\
\text { emocional da equipe de } \\
\text { enfermagem diante da } \\
\text { pandemia de COVID-19: } \\
\text { relato de experiência. }\end{array}$ & $\begin{array}{l}\text { O sentimento de impotência e desgaste emocional causado } \\
\text { pela falta de colaboração dos pacientes no que diz respei- } \\
\text { to às medidas de prevenção do alastramento da doença, } \\
\text { associada a fatores como, falta de treinamento eficiente, } \\
\text { insuficiência de equipamentos de proteção individual e o } \\
\text { medo de contaminar-se ou de contaminar a própria familia, } \\
\text { vem desencadeando doenças mentais preexistentes. }\end{array}$ & REAS. 2020 \\
\hline
\end{tabular}




\begin{tabular}{|c|c|c|c|c|c|}
\hline 05 & $\begin{array}{c}\text { Barbosa DJ, Gomes } \\
\text { MP, Souza FBA, To- } \\
\text { soli Gomes AM. (10) }\end{array}$ & $\begin{array}{c}\text { Fatores de estresse nos } \\
\text { profissionais de enfer- } \\
\text { magem no combate à } \\
\text { pandemia da COVID-19: } \\
\text { Síntese de Evidências }\end{array}$ & $\begin{array}{c}\text { Ameaça não só a saúde física das pessoas, mas também } \\
\text { sua saúde mental, especialmente em termos de emoção e } \\
\text { cognição. }\end{array}$ & $\begin{array}{c}\text { Comunicação } \\
\text { em Ciências da } \\
\text { Saúde. } 2020\end{array}$ \\
\hline 06 & $\begin{array}{c}\text { Bannwart OI, Vieira } \\
\text { MPM, Trindade } \\
\text { LJM, Teodoro SNG, } \\
\text { Vieira FSF. (11) }\end{array}$ & $\begin{array}{c}\text { A saúde mental dos pro- } \\
\text { fissionais de enfermagem } \\
\text { no contexto da pandemia } \\
\text { do novo Coronavírus: } \\
\text { Uma revisão sistemática }\end{array}$ & $\begin{array}{c}\text { Entre os profissionais de enfermagem, os fatores desen- } \\
\text { cadeantes do desequilíbrio da saúde mental podem estar } \\
\text { relacionados ao processo de trabalho, como o turno, o } \\
\text { relacionamento entre a equipe de trabalho, o paciente, os } \\
\text { familiares, a sobrecarga de trabalho, o desgaste, a falta } \\
\text { de equipamentos de proteção individual (EPI's), o suporte } \\
\text { social, o conflito de interesses e as estratégias de enfrenta- } \\
\text { mento desenvolvidas para evitar contaminação. }\end{array}$ & $\begin{array}{c}\text { Revista Cientí- } \\
\text { fica Cognitionis. } \\
2020\end{array}$ \\
\hline
\end{tabular}

\section{DISCUSSÃO}

Os profissionais da enfermagem são responsáveis pelo gerenciamento de equipes de enfermagem e materiais na assistência em hospitais, promovendo cuidados integrais aos pacientes. E no contexto da pandemia não seria diferente, eles continuam na linha de frente lutando contra um inimigo invisível denominado Coronavírus, que tem conservado a sociedade enclausurada, mas esses profissionais ao contrário do enclausuramento, estão cada dia mais expostos e trabalhando na luta diária para salvar vidas. Observando este cenário, é possível constatar que a pandemia do COVID-19 tem sido uma fonte de grande estresse, visto que, diferentes indivíduos experimentam distintos níveis de crises emocionais. Entretanto, é especialmente mais difícil para aqueles que estão atuando no centro da crise ${ }^{(11,12)}$.

Nesse cenário, medo, ansiedade, depressão, (entre outros transtornos) são alguns problemas que podem prejudicar psicológica e emocionalmente o trabalho dos profissionais de enfermagem, fazendo com que o enfrentamento físico e mental seja comprometido, o que gera a ineficácia da produtividade do trabalho, aliada ao aumento do índice de acidentes, assistência comprometida, o que nas condições de uma pandemia, não deve existir, visto que resulta em prejuízos e afeta a segurança dos pacientes e a qualidade do trabalho ${ }^{(3)}$.

A enfermagem compreende a maior categoria profissional dos serviços de saúde mundial, estando diretamente ligada aos cuidados com pacientes portadores do vírus. Diante disso, estão mais propícios e vulneráveis a adquirir o vírus e desenvolver impactos psicológicos, afinal, a situação pandêmica gera estresse, medo, angústia e entre outros sentimentos negativos ao bem-estar da saúde emocional, que quando não tratados, pode evoluir para quadros mais severos, como a depressão crônica. Vale ressaltar, que alterações emocionais negativas na enfermagem, pode comprometer a qualidade e a eficiência dos cuidados em saúde destinados à população ${ }^{(6)}$.

Desde o início do surto pandêmico de COVID-19, inúmeros profissionais de enfermagem foram infectados pelo vírus, apresentaram a forma mais branda ou evoluíram para forma mais severa, levando a óbitos. Frente a esse panorama, tais profissionais expõem-se a desgastes emocionais constantes, pois além da preocupação com a assistência e risco de contaminação, ainda enfrentam a escassez de equipamentos de proteção individual e falta de treinamentos eficientes, principalmente quanto ao manejo com o paciente e a utilização correta do EPI, o que agrava ainda mais o seu estado emocional ${ }^{(9)}$.

Um dos pontos que mais preocuparam os profissionais da linha de frente do enfrentamento ao covid-19, é que além da apreensão para conservar a vida e a saúde, ainda pesam sobre eles o medo de infectar seus familiares, por isso, muitos acabaram optando por isolar-se por completo do convívio familiar ${ }^{(9)}$. O elevado número de óbitos de colegas de trabalho e de pacientes causado pelo vírus ocasiona um grande risco psicopatológico aos profissionais que atuam na linha de frente, pois desperta angústia e ansiedade, e reforça o sentimento de impotência diante do cenário vivido ${ }^{(9,10)}$.

A COVID-19 se trata de uma doença com alto grau de transmissibilidade entre os indivíduos, podendo estes serem assintomáticos ou sintomáticos. Por causa desse estado de calamidade global gerado pela pandemia, os agravos da saúde mental como o estresse entre outros, faz com que os enfermeiros, vivenciem uma rotina de medo e incertezas. O meio hospitalar exige do enfermeiro, atividades intensas, o lidar com a dor, sofrimento, morte e recuperações, favorecendo a manifestação de oscilações e abalos emocionais ${ }^{(7)}$.

Atualmente, o profissional enfermeiro tem que combater desafios impostos pela pandemia do COVID-19, como o alto risco de contaminação, o adoecimento que pode desencadear em angústia, fadiga, sentimento de incapacidade por não conseguir salvar vidas, ameaças, ofensas e distanciamento da sociedade e elevado índice de óbitos. Todos esses desgastes, comprometem a saúde física e mental do trabalhador causando assim somatizações, que também impactam na qualidade do atendimento prestado ao paciente ${ }^{(7)}$.

Estão atrelados a isso, os impactos físicos e psíquicos à saúde do trabalhador, podendo resultar em perturbações emocionais, que quando associadas à 


\section{artigo}

Costa, L.E.S.; Oliveira, F.V.; Carneiro, A.G.M.; Ribeiro, S.A.A.; Barros, A.C.S.; Tavares, P.P.C.

Repercussões psicopatológicas em enfermagem decorrentes da pandemia do COVID-19: uma revisão integrativa

desvalorização, baixa remuneração e outras condições desfavoráveis de trabalho, desencadeiam psicopatologias como a síndrome de Burnout, caracterizada pelo desgaste físico e psíquico de profissionais que exercem suas funções, depressão e até mesmo, o suicídio ${ }^{(7)}$.

Salienta-se que a enfermagem exerce um papel fundamental nas diversas áreas de combate ao COVID-19, tais como prevenção, mitigação e combate, no entanto este profissional precisa ter condições laborais apropriadas para desenvolver seu trabalho de forma segura, carecendo de acompanhamento psicológico em decorrência da quantidade de vidas perdidas em um curto espaço de tempo, se assim não ocorrer, o mesmo pode desenvolver diversas psicopatologias, decorrentes do medo de ser exposto ao vírus, da sobrecarga de trabalho, desvalorização e do medo de estar transmitindo esse vírus para seus amigos, conhecidos e familiares ${ }^{(8)}$.

A atual situação sanitária do brasil evidencia o estado de vulnerabilidade que a enfermagem está sendo exposta e condicionada, principalmente no que se refere aos profissionais atuantes em unidades hospitalares que são submetidos a trabalho exaustivo o que resulta em desequilíbrio na relação demanda-profissional. E nesse cenário de atuação, destaque, risco de contaminação, somatizações devido a medo, estresse e desgaste físico e emocional salienta-se que apesar da enfermagem ser a arte do cuidar, faz necessário que a profissão e profissionais sejam cuidados e valorizados para que assim o equilíbrio assistencial na saúde seja mantido. ${ }^{(4)}$

\section{CONCLUSÃO}

Diante dos achados, foi possível identificar que os profissionais de enfermagem na linha de frente, diretamente envolvidos no diagnóstico, tratamento e cuidado de pacientes com COVID-19, correm sérios riscos de desenvolver sofrimento psicopatológico, comprometendo assim a saúde mental e por consequência as próprias vidas, bem como a assistência prestada aos pacientes. Por essa razão, é importante oferecer suporte profissional especializado aos profissionais de enfermagem, por meio de equipe multiprofissional garantindo melhores condições de trabalho e apoio aos membros da equipe de enfermagem, favorecendo processos assistenciais melhorados.

\section{REFERÊNCIAS}

1. Organização Mundial de Saúde. OMS afirma que COVID-19 é agora caracterizada como pandemia [Internet]. Brasilia; 2020 [cited 2020 Dec 16]. Available from: https://www. paho.org/bra/index.php?option=com_content\&view=article\&id=6120:oms-afirma-que-covid-19-e-agora-caracterizada-como-pandemia\&Itemid=812

2. Organização Mundial de Saúde. Infográfico: Fotografia da Enfermagem no Brasil [Internet]. Washington; 2020 [cited 2020 Dec 16]. Available from: https://www. paho.org/bra/index.php?option=com_content\&view=article\&id=6120:oms-afirma-que-covid-19-e-agora-caracterizada-como-pandemia\&Itemid $=812$

3. Dal'Bosco EB, Floriano LSM, Skupien SV, Arcaro G, Martins AR, Anselmo CC. Saúde mental da enfermagem no enfrentamento do COVID-19 em um hospital universitário regional. Rev. Bras. Enferm. 2020; 73:1-7.

4. Luna Filha DOM, Magalhães BC, Silva MMO, Albuquerque GA. Cuidamos dos outros, mas quem cuida de Nós? Vulnerabilidades e implicações da COVID-19 na enfermagem. Enferm. Foco 2020; 11 (1) Especial: 135-140

5. Huang C, Wang Y, Li X, Ren L, Zhao J, Hu Y, et al. Clinical features of patients infected with 2019 novel coronavirus in Wuhan, China. The Lancet, 2020; V.395: 497-506.

6. Ramos-Toescher AM, Tomaschewisk-Barlem JG, Barlem ELD, Castanheira JS, Toescher RL. Saúde mental de profissionais da enfermagem durante a pandemia da COVID-19: recursos de apoio. Esc. Ana Nery. 2020; 24(1):1-7.

7. Pereira MD, Torres EC, Pereira MD, Antunes PFS, Costa CFT. Sofrimento emocional dos enfermeiros no contexto hospitalar frente à pandemia de COVID-19. Research, Society and Development. 2020; 9(8): 1-21

8. Souza NVDO, Carvalho EC, Soares SSS, Varella TCMM, Pereira SEM, Andrade KBS. Trabalho de enfermagem na pandemia da Covid-19 e repercussões para a saúde mental dos trabalhadores. Rev Gaúcha Enferm. 2021; 021;42(esp): $1-13$.

9. Portugal JKA, Reis MHS, Barão Évelyn JS, Souza TTG, Guimarães RS, Almeida LS, et al. Percepção do impacto emocional da equipe de enfermagem diante da pandemia de COVID-19: relato de experiência. REAS [Internet]. 21 maio2020 [citado 28jan.2021];(46):e3794. Available from: https://acervomais.com.br/index.php/saude/article/ view/3794

10. Barbosa DJ, Pereira Gomes M, Barbosa Assumpção de Souza F, Tosoli Gomes AM. Fatores de estresse nos profissionais de enfermagem no combate à pandemia da COVID-19: síntese de evidências. Com. Ciências Saúde [Internet]. $5^{\circ}$ de maio de 2020 [citado $28^{\circ}$ de janeiro de 2021];31(Suppl 1):31-47. Disponível em: http://www.escs. edu.br/revistaccs/index.php/comunicacaoemcienciasdasaude/article/view/651

11. Bannwart OI, Vieira MPM, Trindade LJM, Teodoro SNG, Vieira FSF. A saúde mental dos profissionais de enfermagem no contexto da pandemia do novo Coronavírus: Uma revisão sistemática. Revista Científica Cognitionis, 2020; 2(2): 1-10.

12. Teixeira CFS, Soares CM, Souza EA, et al. A saúde dos profissionais de saúde no enfrentamento da pandemia de Covid-19. Ciênc. saúde coletiva. 2020; 25:3465- 